\title{
Molecular identification of adenovirus causing respiratory tract infection in pediatric patients at the University of Malaya Medical Center
}

\author{
Juraina Abd-Jamil†, Boon-Teong Teoh†, Eddy H Hassan`, Nuruliza Roslan` and Sazaly AbuBakar*†
}

\begin{abstract}
Background: There are at least 51 adenovirus serotypes (AdV) known to cause human infections. The prevalence of the different human AdV (HAdV) serotypes varies among different regions. Presently, there are no reports of the prevalent HAdV types found in Malaysia. The present study was undertaken to identify the HAdV types associated primarily with respiratory tract infections (RTI) of young children in Malaysia.

Methods: Archived HAdV isolates from pediatric patients with RTI seen at the University of Malaya Medical Center (UMMC), Kuala Lumpur, Malaysia from 1999 to 2005 were used. Virus isolates were inoculated into cell culture and DNA was extracted when cells showed significant cytopathic effects. AdV partial hexon gene was amplified and the sequences together with other known HAdV hexon gene sequences were used to build phylogenetic trees. Identification of HAdV types found among young children in Malaysia was inferred from the phylograms.

Results: At least 2,583 pediatric patients with RTI sought consultation and treatment at the UMMC from 1999 to 2005. Among these patients, $48(<2 \%)$ were positive for HAdV infections. Twenty-seven isolates were recovered and used for the present study. Nineteen of the 27 ( 70\%) isolates belonged to HAdV species C (HAdV-C) and six ( 22\%) were of HAdV species B (HAdV-B). Among the HAdV-C species, 14 ( $74 \%$ ) of them were identified as HAdV type 1 (HAdV-1) and HAdV type 2 (HAdV-2), and among the HAdV-B species, HAdV type 3 (HAdV-3) was the most common serotype identified. HAdV-C species also was isolated from throat and rectal swabs of children with hand, foot, and mouth disease (HFMD). Two isolates were identified as corresponding to HAdV-F species from a child with HFMD and a patient with intestinal obstruction.

Conclusions: HAdV-1 and HAdV-2 were the most common HAdV isolated from pediatric patients who sought treatment for RTI at the UMMC from 1999 to 2005. HAdV-B, mainly HAdV-3, was recovered from $\sim 22 \%$ of the patients. These findings provide a benchmark for future studies on the prevalence and epidemiology of HAdV types in Malaysia and in the region.
\end{abstract}

\section{Background}

Respiratory tract infections (RTI) are common in adults and children worldwide. The disease varies in severity, presenting as uncomplicated, subacute, acute and chronic infection. RTIs can be life threatening depending on the causative agent and host condition. In children, a high incidence of RTI is caused either by: 1) heightened expo-

* Correspondence: sazaly@um.edu.my

1 Tropical Infectious Diseases Research and Education Center (TIDREC), Department of Medical Microbiology, Faculty of Medicine, University of

Malaya, 50603 Kuala Lumpur, Malaysia

+ Contributed equally

Full list of author information is available at the end of the article sure of young children to RTI infectious agents from siblings, friends, and child care; 2) environmental factors; and 3) inherited disorders of the immune system [1]. In industrialized and developed countries, nearly $50 \%$ of pediatric consultations are RTI related [2,3], and at least 1.9 million children died from acute RTI with $70 \%$ of them in Africa and Asia in 2000 [4].

There are a number of infectious agents that cause RTI. Bacteria, such as Haemophilus influenzae, Streptococcus pneumoniae, Escherichia coli, Klebsiella pneumoniae, Mycoplasma pneumonia, and Chlamydia trachomatis are among the most common. However, $80 \%-90 \%$ RTI are 
caused by viruses, such as respiratory syncytial virus (RSV), influenza virus, parainfluenza virus, and adenovirus (AdV) [1,5]. RSV is one of the most common agents of RTI in infants and young children in many countries. It is estimated to cause $~ 39 \%$ of all pneumonia cases and up to $6 \%$ of pneumonia-associated deaths [6]. Parainfluenza virus and influenza virus also are commonly isolated viruses from children with viral RTI.

HAdV-associated RTI is reportedly low. It accounts for $4 \%-10 \%$ cases of pneumonia, $2 \%-10 \%$ cases of bronchiolitis, and 3\%-9\% cases of croup [7]. Severity of infection associated with HAdV varies with the different HAdV serotypes [7-9]. There are at least 51 immunologically distinct HAdV types classified into six species, designated A to F [10]. Viruses causing RTI are usually isolated in the laboratory from patients' nasal secretions and serotyped by immunological typing methods [11-13]. In recent years, molecular identification of the virus has become more common, where distinction of the virus types can be made through specific genomic sequence amplification by polymerase chain reaction (PCR) and determination of the partial hexon gene sequence $[14,15]$. Presently, there is no report of the prevalent HAdV types causing infections among Malaysians. This could be due to the low infection and fatality rates of the infection resulting in limited interest in typing the virus. The present study was undertaken to type HAdV of pediatric patients younger than 5 years seen at the University of Malaya Medical Center (UMMC), Kuala Lumpur, Malaysia.

\section{Methods}

\section{Virus}

Twenty-seven archived HAdV isolates were recovered from the UMMC virology repository and used for the study. The isolates were derived mainly from the nasopharyngeal secretions (NPS) of children younger than 5 years diagnosed with RTI (Table 1). Virus isolation and propagation were performed using African green monkey kidney cells (Vero), human lung carcinoma cells (A549), and dog kidney cells (MDCK). The presence of HAdV was detected by immunofluorescence staining using specific antibodies (Cat. No. 5000; Light Diagnostics Inc., Salt Lake City, UT, USA) following manifestation of cytopathic effects. Virus inoculum was prepared and kept at $-70^{\circ} \mathrm{C}$ until needed for genomic typing. Further maintenance and propagation of the HAdV isolates were performed in A549 cells.

\section{PCR amplification and genome sequencing}

Viral genomic DNA was extracted using Tri Reagent (Molecular Research Center Inc., Cincinnati, OH, USA) following the manufacturer's protocol. The partial hexon gene was amplified using the primer pair, AdTU7 (5'-
GCCACCTTCTTCCCCATGGC-3') and AdTU4 (5'GTAGCGTTGCCGGCCGAGAA-3') to amplify a 1,001 bp fragment of the partial hexon gene (position 20,733 21,734; Accession \# NC_001405). A nested polymerase chain reaction (PCR) also was performed on the amplified fragment using the primers, AdU-S (5'-TTCCCCATGGCNCACAACAC-3') and AdU-A (5'-GCCT CGATGACGCCGCGGTG-3') which resulted in a 956 bp fragment. Amplification was performed for 36 cycles consisting of a denaturation step at $94^{\circ} \mathrm{C}$ for $1 \mathrm{~min}$, an annealing step at $50^{\circ} \mathrm{C}$ for $1 \mathrm{~min}$, and an extension step at $72^{\circ} \mathrm{C}$ for $2 \mathrm{~min}$. The extension was continued at $72^{\circ} \mathrm{C}$ for 7 min. The amplified DNA fragment was separated in 1.5\% agarose gel (Promega, Madison, WI, USA), and purified using QIA Quick gel extraction system (Qiagen $\mathrm{GmbH}$, Hilden, Germany) according to the manufacturer's protocol. The DNA fragments were sequenced at Macrogen Inc. (Seoul, Korea).

\section{Sequence and phylogenetic analysis}

The partial hexon gene sequences were aligned and phylogenetic trees were drawn as previously described [16]. Briefly, the HAdV partial hexon gene sequences were analyzed using Sequencher 4.6 (Gene Codes Corporation, Ann Arbor, MI) and aligned against other available AdV sequences using ClustalX [17]. Phylogenetic trees were drawn using the maximum-likelihood method as implemented in PHYLIP 3.67 [18] and the maximumparsimony method using MEGA4 [19]. Bootstrap values were obtained from a random sampling of 100 replicates. Reference HAdV sequences used to build the phylogenetic trees were obtained from the GenBank. Details on the reference sequences are shown in Table 2.

The study was approved by the University Malaya Medical Centre Ethics Committee (Approval \#794.6).

\section{Results}

A total of 2,583 pediatric patients with RTI were treated at the UMMC from 1999 to 2005 . Of these patients, 48 $(<2 \%)$ were positive for HAdV by either direct immunofluorescence staining of the patient's NPS, PCR amplification, or virus isolation. HAdV also was isolated from the throats of patients with HFMD. These HFMD patients usually did not present with RTI symptoms, but throat swabs were routinely collected in addition to rectal swabs.

In the present study, the partial hexon gene sequences (nucleotide 20,734-21,737) of 27 HAdV isolates from UMMC were determined. The sequences were aligned and phylogenetic trees were drawn using the maximumlikelihood and maximum-parsimony methods. Only results from the maximum-likelihood method were presented, as trees drawn from both the methods were similar. The nucleotide sequence alignment clustered the 
Table 1: Human adenoviruses isolated at the University of Malaya Medical Centre from 1999 to 2005.

\begin{tabular}{|c|c|c|c|c|c|}
\hline Sample & Year & Isolation site ${ }^{a}$ & Age & Diagnosis & Serotype \\
\hline AD01MY99 & 1999 & NPS & $N A^{b}$ & Pneumonia & B3 \\
\hline AD02MY99 & 1999 & NPS & $3 \mathrm{mth}$ & Chronic lung diseases & B7 \\
\hline AD04MY00 & 2000 & RS & $7 \mathrm{mth}$ & HFMD & F40 \\
\hline AD05MY00 & 2000 & TS & $1 \mathrm{yr}$ & HFMD & $\mathrm{C} 2$ \\
\hline AD06MY00 & 2000 & TS & $8 \mathrm{mth}$ & HFMD & $\mathrm{C} 1$ \\
\hline AD07MY01 & 2001 & RS & $6 \mathrm{mth}$ & Intestinal obstruction & F41 \\
\hline AD08MY00 & 2000 & RS & NA & HFMD & $\mathrm{C} 5$ \\
\hline AD09MY01 & 2001 & NPS & $\mathrm{lyr}$ & Acute bronchiolitis & $\mathrm{C} 1$ \\
\hline AD10MY01 & 2001 & NPA & $1 \mathrm{yr}$ & Bronchiolitis & $\mathrm{C} 2$ \\
\hline AD11MY02 & 2002 & NPS & $1 \mathrm{yr}$ & Bronchopnuemonia, hypochronic anemia & $\mathrm{C} 1$ \\
\hline AD12MY02 & 2002 & NPS & $1 \mathrm{yr}$ & Bronchiolitis & $\mathrm{C} 1$ \\
\hline AD13MY03 & 2003 & NPS & $10 \mathrm{mth}$ & Acute gastroenteritis & $\mathrm{C} 2$ \\
\hline AD14MY03 & 2003 & NPS & $1 \mathrm{yr}$ & Viral fever & $\mathrm{C} 5$ \\
\hline AD15MY03 & 2003 & NPS & $8 \mathrm{mth}$ & Acute bronchiolitis & $\mathrm{C} 2$ \\
\hline AD16MY03 & 2003 & NPS & NA & Bronchopneumonia & $\mathrm{C} 2$ \\
\hline AD17MY04 & 2004 & NPS & $2 \mathrm{yr}$ & Acute bronchiolitis & $\mathrm{C} 1$ \\
\hline AD18MY04 & 2004 & NPS & 9 mth & Pneumonia & $\mathrm{C} 5$ \\
\hline AD19MY04 & 2004 & NPS & $5 \mathrm{mth}$ & Bronchiolitis & B3 \\
\hline AD20MY04 & 2004 & NPS & $2 \mathrm{yr}$ & Pneumonia & $\mathrm{C} 2$ \\
\hline AD21MY04 & 2004 & NPS & $3 y r$ & Pneumonia & $\mathrm{C} 1$ \\
\hline AD22MY04 & 2004 & NPS & $1 \mathrm{yr}$ & Bronchopneumonia & $\mathrm{C} 2$ \\
\hline AD23MY04 & 2004 & NPS & $5 \mathrm{mth}$ & Bronchiolitis & $\mathrm{C} 6$ \\
\hline AD24MY04 & 2004 & NPS & $3 \mathrm{yr}$ & Viral fever & B3 \\
\hline AD25MY04 & 2004 & NPS & $8 \mathrm{mth}$ & Pulmonary collapse & $\mathrm{C} 5$ \\
\hline
\end{tabular}


Table 1: Human adenoviruses isolated at the University of Malaya Medical Centre from 1999 to 2005. (Continued)

\begin{tabular}{lcllll}
\hline AD26MY04 & 2004 & NPS & $1 \mathrm{yr}$ & Pneumonia & B3 \\
\hline AD27MY04 & 2004 & NPS & $3 \mathrm{mth}$ & Acute bronchiolitis & C2 \\
\hline AD28MY05 & 2005 & NPS & $2 \mathrm{yr}$ & Bronchopneumonia & B3 \\
\hline $\begin{array}{l}\text { aNPS is nasopharyngeal secretion; RS is rectal swab; TS is throat swab } \\
\text { bNot available }\end{array}$ & & &
\end{tabular}

UMMC isolates into HAdV-C species $(\mathrm{n}=19)$, HAdV-B species $(n=6)$, and HAdV-F species $(n=2)$ with high bootstrap values. Within the HAdV-C species, eight isolates were $\mathrm{HAdV}-2$ (42\%); six isolates, HAdV-1 (32\%); four isolates, HAdV-5 (21\%); and one isolate, HAdV-6 (5\%; Figure 1a). When compared with other HAdV from the GenBank, most of the UMMC HAdV-5 and HAdV-2 isolates formed a separate subcluster of their own (Figure 1a). Within HAdV-B species, five isolates grouped into HAdV-3, and one was HAdV-7 (Figure 1b). HAdV-3 isolates from the study formed a separate subcluster together with an isolate from Guangzhou, China (Accession \# AY878716) distinct from other known HAdV-3. This raised the possibility that the viruses may share a common ancestral lineage with the Malaysian isolate that was isolated in 1999. The HAdV-7 clustered together with isolates from the East Asian countries suggesting a possible widespread presence of the virus in the East Asian region. Four of the HAdV isolates sequenced in the study were from HFMD patients, and three of them clustered together with the $\mathrm{HAdV}-\mathrm{C}$ species. One isolate clustered with known HAdV-40, whereas an isolate from the rectal swab of a patient diagnosed with intestinal obstruction clustered with known HAdV-41. No HAdV$B$ genotype previously associated with fatal cases of HFMD $[20,21]$ was detected in any of the HFMD patient samples. Isolates from HFMD patients were included in the study initially because no effort was made to discriminate the samples from those strictly with RTI. Patients with HFMD normally would not present with RTI symptoms.

\section{Discussion}

Human adenovirus is most commonly associated with respiratory illnesses. However, depending on the infecting serotype, the virus also causes various other illnesses, including gastroenteritis, conjunctivitis, cystitis, and non- specific exanthemas $[13,22]$. Symptoms of the respiratory illness associated with HAdV range from mild infection to severe pneumonia $[8,23]$. Young children and immunocompromised patients are especially vulnerable to severe complications of HAdV infection [24,25]. The findings that less that $2 \%$ of UMMC pediatric RTI is asso- ciated with HAdV respiratory infection is consistent with other reports that $\mathrm{HAdV}$-associated respiratory infection is usually low in comparison to other viruses, such as RSV and parainfluenza virus. The infection also is generally milder and rarely leads to severe complications and deaths $[8,26]$. The low number of HAdV isolation among pediatric patients seen at the UMMC also suggests that the virus is not associated with any major RTI outbreaks during the period from 1999 to 2005. This is perhaps among the reasons why there have not been many efforts to identify the HAdV species and types in children with RTI in many countries, including Malaysia. In addition, the low incidence of RTI caused by HAdV in the community hampered the effort to get enough representative isolates.

In our study, HAdV partial hexon gene sequences were used to type the different HAdV isolates. This gene region contains the hypervariable region that confers HAdV serotype specificity. Using this molecular typing method, HAdV-C species, specifically type 1 and 2, were the most common $\mathrm{HAdV}$ isolated from the pediatric patients seen at UMMC from 1999 to 2005. In contrast, studies done in the United States of America, United Kingdom, Korea, and China, showed HAdV-B species as the most commonly isolated HAdV [27-30]. The reasons for the marked differences are not known. It could be that $\mathrm{HAdV}-\mathrm{C}$ is more common in the region in comparison to the more developed countries. However, the prevalence of HAdV-C species in the neighboring countries could not be compared because information from these countries are lacking.

Overrepresentation of $\mathrm{HAdV}-\mathrm{C}$ in UMMC pediatric patients could suggest a high prevalence of the virus in the community. There are reports that the virus could persist and cause asymptomatic latent infection in rabbits for as long as one year [31]. HAdV-C serotypes 1,2 , and 5 are the most common serotype of HAdV associated in latent infection of tonsils and adenoids of humans, which at times cause RTI in young children [32]. The prolonged presence of the virus in infected children increases its transmissibility, and this could contribute to the persistence of the virus of young children in Malaysia. The ubiquitous presence of the virus also could help explain 
Table 2: Reference adenovirus (AdV) strains from the Genbank used for the typing of human AdV isolated at the University of Malaya Medical Centre.

\begin{tabular}{|c|c|c|}
\hline Accession Number & Serotype & Country/Strain \\
\hline HAdV_A_NC 001460 & $\mathrm{~A} 12$ & ATCC \\
\hline HAdV_B_NC 004001 & B11 & Slobitski \\
\hline HAdV_C_NC 001405 & $\mathrm{C} 2$ & $N A^{a}$ \\
\hline HAdV_D_NC 002067 & D17 & NA \\
\hline HAdV_E_NC 003266 & E4 & Vaccine CL68578 \\
\hline HAdV_F_NC 001454 & $\mathrm{~F} 40$ & Dugan \\
\hline HAdV_F_L19443 & $\mathrm{F} 40$ & Dugan \\
\hline HAdV_3_AY878716 & B3 & Guangzhou, China \\
\hline HAdV_3_AF542108 & B3 & Korea \\
\hline HAdV_3_AF542129 & B3 & Korea \\
\hline HAdV_3_DQ086466 & B3 & NA \\
\hline HAdV_7_AF053085 & B7 & S-1058; Japan \\
\hline HAdV_7_AY769945 & B7 & 95-81; Korea \\
\hline HAdV_7_AF515814 & B7 & China \\
\hline HAdV_7_AC 000018 & B7 & NA \\
\hline HAdV_7_AY769946 & B7 & Strain 99-95; Korea \\
\hline HAdV_7_AB243009 & B7 & Kyoto, Japan \\
\hline HAdV_11_AC 000015 & B11 & NA \\
\hline HAdV_11_AF532578 & B11 & Slobitski \\
\hline HAdV_11_AY163756 & B11 & Ad11p Slobitski \\
\hline HAdV_14_AY803294 & B14 & De Wit/ATCC VR1091 \\
\hline HAdV_14_DQ149612 & B14 & NA \\
\hline HAdV_16_X74662 & B16 & ATCC CH.79 \\
\hline HAdV_21_AB053166 & B21 & AV-1645 \\
\hline
\end{tabular}

Table 2: Reference adenovirus (AdV) strains from the Genbank used for the typing of human AdV isolated at the University of Malaya Medical Centre. (Continued)

\begin{tabular}{|c|c|c|}
\hline HAdV_34_AY737797 & B34 & Compton/ATCC VR716 \\
\hline HAdV_34_AB052911 & B34 & NA \\
\hline HAdV_35_AC 000019 & B35 & Old world monkey strain \\
\hline HAdV_35_AY128640 & B35 & Holden/ATCC VR718 \\
\hline HAdV_35_AY271307 & B35 & $35 p$ \\
\hline HAdV_35_AB052912 & B35 & Japan \\
\hline HAdV_50_DQ149643 & B50 & NA \\
\hline HAdV_50_AY737798 & B50 & Wan/ATCC VR1502 \\
\hline HAdV_1_ AC 000017 & $\mathrm{C} 1$ & P1 C124G1 \\
\hline HAdV_1_Y17244 & $\mathrm{C} 1$ & Ad71 \\
\hline HAdV_2_AC 000007 & $\mathrm{C} 2$ & NA \\
\hline HAdV_2_AY224392 & $\mathrm{C} 2$ & Korea \\
\hline HAdV_2_AF542118 & $\mathrm{C} 2$ & Korea \\
\hline HAdV_2_AJ293905 & $\mathrm{C} 2$ & Germany \\
\hline HAdV_2_AJ293901 & $\mathrm{C} 2$ & United Kingdom \\
\hline HAdV_5_AF542130 & C5 & Korea \\
\hline HAdV_5_AF542128 & $\mathrm{C} 5$ & Korea \\
\hline HAdV_5_AF542124 & C5 & Korea \\
\hline HAdV_5_AC 000008 & $\mathrm{C} 5$ & NA \\
\hline HAdV_5_AF542109 & C5 & Korea \\
\hline HAdV_6_DQ149613 & C6 & NA \\
\hline HAdV_6_Y17245 & C6 & Ton66 \\
\hline HAdV_40_X51782 & $\mathrm{F} 40$ & Dugan \\
\hline HAdV_41_X51783 & F41 & Tak \\
\hline HAdV_41_D13781 & F41 & Tak \\
\hline
\end{tabular}


(a)

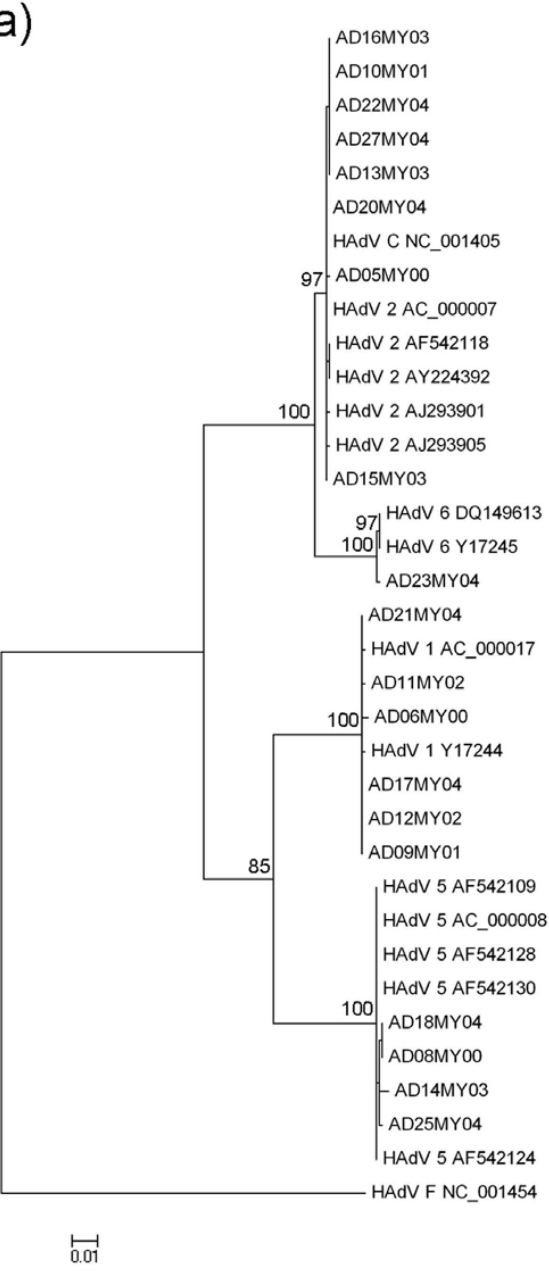

(b)

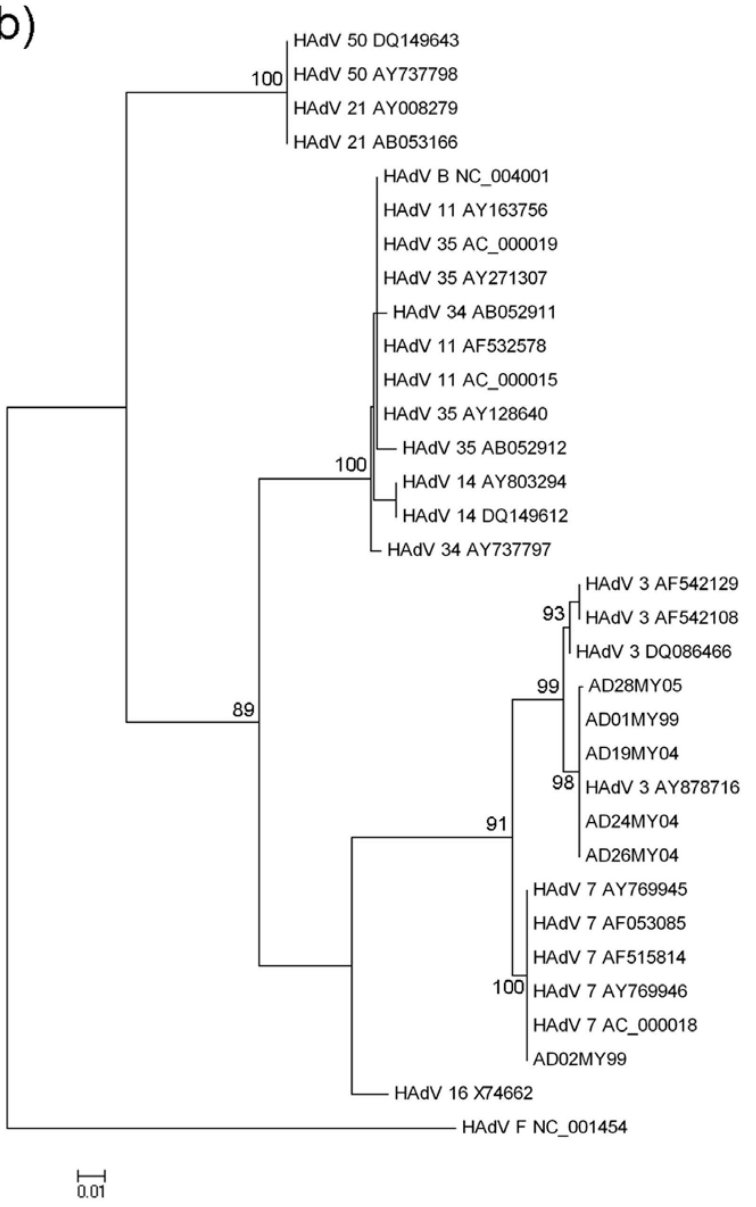

(c)

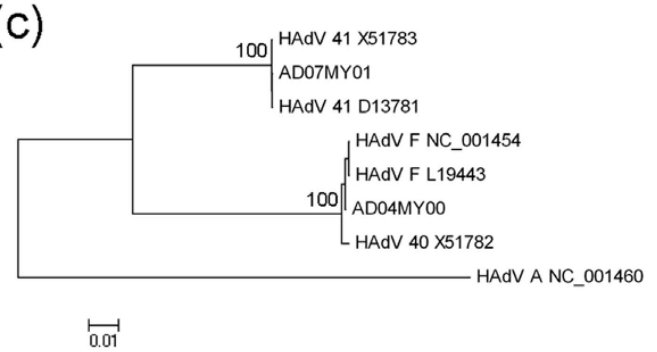

Figure 1 Molecular typing of human adenoviruses (HAdV) isolated at the University of Malaya Medical Center (UMMC) from 1999 to 2005. HAdV-1 and HAdV-2 comprised most of the HAdV-C species isolated at the UMMC (a). HAdV-B species consisted of HAdV-3 and HAdV-7. HAdV-3 formed its own distinct cluster separate from the rest of the group (b). Two isolates from the study were grouped as HAdV-F serotype 40 and 41 (c), commonly implicated in gastroenteritis.

isolation of the virus from patients with HFMD and nonspecific viral fever. On the other hand, the results also could reflect the higher tendency of children with HAdV$\mathrm{C}$ species infection to seek medical attention, hence suggesting that the virus could cause more severe manifestations of RTI. Further studies, however, will be needed to verify this.

\section{Conclusions}

The present study is the first to report the prevalent and circulating HAdV types in Malaysia. It showed that HAdV-C species especially HAdV-1 and HAdV-2 were the most commonly isolated HAdV among pediatric patients seen at UMMC from 1999 to 2005, followed by HAdV-B species type 3 . These viruses are common sero- 
types of HAdV causing acute RTI in pediatric patients. Because no such study has ever been reported in Malaysia, the present study provides a benchmark for future studies of HAdV infection in the country.

\section{Abbreviations}

HAdV: human adenovirus; HFMD: hand, foot and mouth disease; NPS nasopharyngeal secretion; RTI: respiratory tract infection; UMMC: University of Malaya Medical Center.

\section{Competing interests}

The authors declare that they have no competing interests.

\section{Authors' contributions}

$S A B$ is the principal investigator of the study. SAB and JAJ designed the study and drafted and wrote the manuscript. NR and $\mathrm{EHH}$ propagated and maintained the virus isolates and performed the initial genomic sequence amplification. BTT performed the sequence alignment, sequence analysis, and treedrawing. All authors have read and approved the final manuscript.

\section{Acknowledgements}

We thank the Department of Medical Microbiology, University of Malaya for allowing the study to be undertaken using virus isolates from the UMMC Virology repository. None of the authors received specific funding for this study.

\section{Author Details}

Tropical Infectious Diseases Research and Education Center (TIDREC), Department of Medical Microbiology, Faculty of Medicine, University of Malaya, 50603 Kuala Lumpur, Malaysia

Received: 17 November 2009 Accepted: 2 July 2010

Published: 2 July 2010

\section{References}

1. Schaad UB: Prevention of paediatric respiratory tract infections: emphasis on the role of OM-85. Eur Respir Rev 2005, 14:74-77.

2. Eggenberger K: Respiratory tract infections most frequently seen in pediatric outpatient care. Ars Medici 1993, 83:24-40.

3. Kvaerner KJ, Nafstad P, Jaakkola JJ: Upper respiratory morbidity in preschool children: a cross sectional study. Arch Otolaryngol Head Neck Surg 2000, 126:1201-1206.

4. Williams BG, Gouws E, Boschi-Pinto C, Bryce J, Dye C: Estimates of worldwide distribution of child deaths from acute respiratory infections. Lancet Infect Dis 2002, 2:25-32.

5. Bakir TMF, Halawani M, Ramia S: Viral aetiology and epidemiology of acute respiratory infections in hospitalized Saudi children. J Trop Pediatrics 1988, 44:100-103.

6. Weber MW, Mulholland EK, Greenwood BM: Respiratory syncytial virus infection in tropical and developing countries. Trop Med International Health 1998, 3:268-280.

7. Cherry JD: Adenoviruses. In Textbook of Pediatric Infectious Diseases 4th edition. Edited by: Feigin RD, Cherry JD. Philadelphia: WB Saunders; 1998:1666-1684.

8. Schmitz H, Wigand R, Heinrich W: Worldwide epidemiology of human adenovirus infections. Am J Epidemiol 1983, 117:455-466.

9. Larranaga C, Kajon A, Villagra E, Avendano LF: Adenovirus surveillance on children hospitalized for acute lower respiratory infections in Chile (1988-1996). J Med Virol 2000, 60:342-346.

10. Shenk T, Horwitz MS: Adenoviridae: the viruses and their replication. In Fields Virology 4th edition. Edited by: Fields BN, Knipe DM, Howley PM. Philadelphia: Lippincott-Raven Publishers; 2001:2265-2326.

11. Hierholzer JC: Adenoviruses. In Diagnostic Procedures for Viral, Rickettsial and Chlamydial Infections 7th edition. Edited by: Lennette EH, Lennette DA, Lennette ET. Washington DC: American Public Health Association: 1995:169-188

12. Hierholzer JC: Further subgrouping of the human adenoviruses by differential agglutination. J Infect Dis 1973, 128:541-550.

13. Wadell G: Molecular epidemiology of human adenoviruses. Curr Trop Microbiol Immunol 1984, 110:191-220.
14. Elnifro EM, Cooper RJ, Klapper PE, Bailey AS: PCR and restriction endonuclease analysis for rapid identification of human adenovirus subgenera. J Clin Microbiol 2000, 38:2055-2061.

15. Casas I, Avellon A, Mosquera M, Jabado O, Echevarria JE, Campos RH, Rewers M, Perez-Brena P, Lipkin WI, Palacios G: Molecular identification of adenoviruses in clinical samples by analyzing a partial hexon genomic region. J Clin Microbiol 2005, 43:6176-6182.

16. AbuBakar S, Wong PF, Chan YF: Emergence of dengue virus type 4 genotype IIA in Malaysia. J Gen Viro/ 2002, 83:2437-2442.

17. Thompson JD, Higgins GD, Gibson TJ: CLUSTAL W: improving the sensitivity of progressive multiple sequence alignments through sequence weighting, position specific gap penalties and weight matrix choice. Nucl Acids Res 1994, 22:4673-4680.

18. Felsenstein J: PHYLIP (Phylogeny Inference Package) version 3.67. Distributed by the author. Department of Genetics, University of Washington, Seattle.

19. Tamura K, Dudley J, Nei M, Kumar S: MEGA4: Molecular Evolutionary Genetics Analysis (MEGA) software version 4.0. Mol Biol Evol 2007, 24:1596-1599.

20. Cardosa MJ, Krishnan S, Tio PH, Perera D, Wong SC: Isolation of subgenus $B$ adenovirus during a fatal outbreak of enterovirus 71-associated hand, foot and mouth disease in Sibu, Sarawak. Lancet 1999, 354:987-991.

21. AbuBakar S, Chee HY, Shafee N: Adenovirus in EV71-associated hand foot and mouth disease. Lancet 2000, 355:416.

22. Wadell G, Allard A, Hierholzer JC: Adenoviruses. In Manual of Clinical Microbiology 7th edition. Edited by: Murray PR, Baron EJ, Pfaller MA, Tenover F, Yolken RN. Washington DC: American Society for Microbiology; 1999:970-982.

23. Horwitz MS: Adenoviruses. In Fields Virology 4th edition. Edited by: Fields BN, Knipe DM, Howley PM, Griffin DE. Philadelphia: Lippincott Williams \& Wilkins; 2001:2301-2326

24. Kojaoghlanian T, Flomenberg P, Horwitz MS: The impact of adenovirus infection on the immunocompromised host. Rev Med Virol 2003, 13:155-171.

25. Walls T, Shankar AG, Shingadia D: Adenovirus: an increasingly important pathogen in paediatric bone marrow transplant patients. Lancet Infect Dis 2003, 3:79-86.

26. Zamberi S, Zulkifli I, llina : Respiratory viruses detected in hospitalised paediatric patients with respiratory infections. Med J Malaysia 2003, 58:681-687.

27. Fox JP, Hall CE, Cooney MK: The Seattle virus watch. IV. Observations of adenovirus infections. Am J Epidemio/ 1977, 105:362-386.

28. Cooper RJ, Hallett R, Tullo AB, Klapper PE: The epidemiology of adenovirus infections in Greater Manchester, UK 1982-96. Epidemiol Infect 2000, 125:333-345.

29. Hong JY, Lee HJ, Piedra PA, Choi EH, Park KH, Koh YY, Kim WS: Lower respiratory tract infections due to adenovirus in hospitalized Korean children: epidemiology, clinical features, and prognosis. Clin Infect Dis 2001, 32:1423-1429.

30. Li QG, Zheng QJ, Liu YH, Wadell G: Molecular epidemiology of adenovirus types 3 and 7 isolated from children with pneumonia in Beijing. J Med Virol 1996, 49:170-177.

31. Reddick RA, Lefkowitz SS: In vitro immune responses of rabbits with persistent adenovirus type 5 infection. J Immunol 1969, 103:687-694.

32. Pereira HG: Persistent infection by adenoviruses. J Clin Patho/ Suppl 1972, 6:39-42

\section{Pre-publication history}

The pre-publication history for this paper can be accessed here: http://www.biomedcentral.com/1471-2431/10/46/prepub

doi: 10.1186/1471-2431-10-46

Cite this article as: Abd-Jamil et al., Molecular identification of adenovirus causing respiratory tract infection in pediatric patients at the University of Malaya Medical Center BMC Pediatrics 2010, 10:46 